SHORT REPORT

\title{
Troponin T: how high is high? Relationship and differences between serum cardiac markers according to level of creatine kinase and type of myocardial infarction
}

\author{
S G Williams, J H Silas, F Joseph, M Jackson, D Rittoo, P Currie
}

See end of article for

Postgrad Med J 2004;80:613-614. doi: 10.1136/pgmj.2003.016253

authors' affiliations

Correspondence to: Dr S G Williams, Cardiothoracic Centre, Thomas Drive, Liverpool L14 3PE, UK;

drsgwilliams@hotmail.com

Submitted

20 October 2003

Accepted 3 January 2004
Cardiac troponins have emerged over recent years as the "gold standard" serum biochemical marker for the diagnosis and management for patients with acute myocardial infarction (MI). The relationship between old (creatine kinase; $\mathrm{CK}$ ) and new (troponin $\mathrm{T} ; \mathrm{T}$ ) markers of myocardial injury were examined in this study of 392 consecutive patients admitted to a district hospital with a diagnosis of an acute Ml. Significant correlation of serum $\Pi$ and peak CK levels were seen $(R=0.58, p<0.0001)$ in all types of Ml. A significant relationship was also seen according to type of $M I$ ( $Q$ wave or non- $Q$ wave $M I)$ or peak CK level. The regression equation $(T T(\mu \mathrm{g} / \mathrm{l})=0.0027$ (peak CK) $+1.1160(\mathrm{IU} / \mathrm{I}))$ may be used by clinicians to estimate $\Pi \pi$ release from a known peak $\mathrm{CK}$ result and thus provide some guidance on equivalence between the two tests. Our findings provide physicians with a benchmark reference range between the two cardiac markers, according to level of peak CK. lis recent years the development of sensitive and specific biochemical markers of myocardial injury (cardiac troponins) has aided the diagnosis and management of acute myocardial infarction (MI). The European Society of Cardiology (ESC) and American College of Cardiology (ACC) have redefined the diagnosis criteria for acute MI to include the measurement of cardiac troponin as the gold standard. ${ }^{1}$ As a result of these recommendations and the increased availability of troponin assays, the diagnosis rate of MI may increase, ${ }^{2}$ which is likely to have an impact on patient management and epidemiology. ${ }^{3}$ We examined the relationship between old (creatine kinase; CK) and new (troponin T; TT) markers of myocardial injury categorised according to electrocardiographic (ECG) changes and peak CK levels, and assessed the differences between the groups. We also aimed to assess the relative value of TT to peak CK in order to provide physicians with a reference range between the two cardiac markers.

\section{METHODS}

We studied 392 consecutive patients (70\% male; mean age 60.5 years, range 32 to 94 ) admitted to a district general hospital in the UK with an acute MI over a six month period (January to June 2002). ECG changes, thrombolysis status, peak serum CK, and TT (12-24 hours following admission) concentrations were recorded. Myocardial infarction was defined according to current ESC/ACC guidelines: a raised serum TT $(\geqslant 0.1 \mu \mathrm{g} / \mathrm{l})$ concentration (in the absence of nonmyocardial causes such as renal failure, thromboembolic disease, or myocarditis) and ECG changes. The study population was then divided into two subgroups for further analysis: (1) according to ECG changes: ST segment elevation/Q wave or non-ST segment elevation/Q wave MI; and (2) according to peak CK levels: levels above or below twice the upper limit of normal (390 IU/l for our laboratory)-that is, the old World Health Organisation biochemical definition of MI. ${ }^{4}$ Patients with peak CK levels > 390 IU/l were classified into further groups for analysis: levels of 390999 IU/l, 1000-1999 IU/l, and >2000 IU/l. The difference in levels of each serum marker between the subgroups was compared using Student's $t$ test for unpaired samples. Values of TT and CK were normalised by logarithmic transformation prior to correlation using Pearson's correlation methods. Simple linear regression was used to derive an equation to facilitate the prediction of TT level from measures of peak CK, and $\mathrm{p}<0.05$ was considered statistically significant.

\section{RESULTS}

Results are summarised and expressed as means (SD) in table 1. A Q wave MI occurred in 286 patients (73\%). Serum TT level in this group was $5.25(6.40) \mu \mathrm{g} / \mathrm{l}$, with a peak CK level of 1379 (1370) IU/l. There was a significant correlation between serum TT and peak CK levels $(R=0.58, \mathrm{p}<0.0001)$. Thrombolysis therapy was given to $185(80 \%)$ patients in this group. Significantly higher levels of TT were observed in the subgroup of patients who underwent thrombolysis than in those who did not $(5.83 \quad(6.73) \mu \mathrm{g} / \mathrm{l} v 22.84$ (3.97) $\mu \mathrm{g} / \mathrm{l}$, $\mathrm{p}<0.0001)$. However, peak CK levels did not significantly differ according to thrombolysis status (1431 (1448) IU/l (thrombolysis) $v 1161 \quad$ (963) IU/l (no thrombolysis), $\mathrm{p}=0.10)$. A non-Q wave MI was found in 106 patients (27\%). Serum TT and peak CK levels were significantly lower in this group than in the Q wave MI group (TT 2.14 (2.07) $v$ 5.25 (6.40) $\mu \mathrm{g} / \mathrm{l}, \mathrm{p}<0.0001$; peak CK 864 (767) $v 1379$ (1370) IU/l, $\mathrm{p}<0.0001$ ). However, a significant correlation between TT and peak CK levels was observed in this subgroup $(R=0.35, \mathrm{p}<0.0001)$.

Overall, 309 patients (79\%) had a peak CK level $>390$ IU/l. Mean TT level in this group was 5.23 (6.10) $\mu \mathrm{g} / \mathrm{l} v 1.48$ (2.29) $\mu \mathrm{g} / \mathrm{l}$ in the group of patients with a peak CK $<390 \mathrm{IU} / \mathrm{l}$, $\mathrm{p}<0.0001)$. A significant correlation was seen between TT and peak CK levels in the patients with peak CK >390 IU/ $(R=0.54, \mathrm{p}<0.0001)$ and to a lesser extent in the group with peak $\mathrm{CK}<390 \mathrm{IU} / \mathrm{l}(R=0.33, \mathrm{p}=0.002)$. As peak CK level increased, the correlation with TT increased, although statistical significance was seen in all peak CK groups (CK 390-999, $R=0.31, \quad \mathrm{p}=0.002 ; \quad \mathrm{CK} 1000-1999, \quad R=0.35$, $\mathrm{p}=0.001 ; \mathrm{CK}>2000, R=0.49, \mathrm{p}<0.0001)$.

Abbreviations: ACC, American College of Cardiology; CK, creatine kinase; ECG, electrocardiographic; ESC, European Society of Cardiology; Ml, myocardial infarction; $\Pi$, troponin T 
Table 1 Correlation between serum $\Pi$ and peak CK levels (mean (SD)) according to type of $\mathrm{Ml}$ and peak CK level

\begin{tabular}{|c|c|c|c|c|}
\hline & $\pi(\mu \mathrm{g} / \mathrm{l})$ & Peak CK (IU/I) & $R$ & $\mathrm{p}$ Value \\
\hline \multicolumn{5}{|l|}{ Type of Ml } \\
\hline $\begin{array}{l}\text { Q wave } \\
\text { (overall) }\end{array}$ & $5.25(6.40)$ & 1379 (1370) & 0.58 & $<0.0001$ \\
\hline $\begin{array}{l}\text { Q wave } \\
\text { (thrombolysis) }\end{array}$ & $5.83(6.73)$ & 1431 (1448) & 0.58 & $<0.0001$ \\
\hline $\begin{array}{l}\text { Q wave (no } \\
\text { thrombolysis) }\end{array}$ & $2.84(3.97)$ & 1161 (963) & 0.55 & $<0.0001$ \\
\hline Non- $Q$ wave & $2.14(2.07)$ & 864 (767) & 0.35 & $<0.0001$ \\
\hline \multicolumn{5}{|l|}{ Peak CK level } \\
\hline$<390(n=83)$ & $1.48(2.29)$ & 207 (112) & 0.33 & 0.002 \\
\hline $\begin{array}{l}>390 \text { (overall, } \\
n=309 \text { ) }\end{array}$ & 5.2316 .100 & $1523(1274)$ & 0.54 & $<0.0001$ \\
\hline $\begin{array}{l}390-999 \\
(n=133)\end{array}$ & $2.55(2.91)$ & $666(186)$ & 0.31 & 0.002 \\
\hline $\begin{array}{l}1000-1999 \\
(\mathrm{n}=111)\end{array}$ & $5.28(5.66)$ & $1396(298)$ & 0.35 & 0.001 \\
\hline $\begin{array}{l}>2000 \\
(n=65)\end{array}$ & $10.59(7.94)$ & 3380 (1527) & 0.49 & $<0.0001$ \\
\hline
\end{tabular}

To allow prediction of TT level from measurement of peak $\mathrm{CK}$, the following regression equation was obtained: $\mathrm{TT}=0.0027$ (peak CK) +1.1160 , SE of the regression slope 0.0002 (95\% confidence interval for population value of slope 0.0023 to 0.0030$)$.

\section{DISCUSSION}

Measurement of biochemical markers of myocardial injury is arguably becoming increasingly important after publication of the ESC/ACC redefinition of MI document. ${ }^{3}$ Our findings provide a benchmark reference range for levels of TT according to peak CK levels. The regression equation may be used by clinicians to estimate TT release from a known peak CK result and thus provide some guidance on equivalence between the two tests. The study shows a significant relationship between serum TT and peak CK concentration in both types of MI (Q or non-Q wave MI) or according to level of peak CK. Serum TT and peak CK levels were significantly higher in the patients who sustained a Q wave rather than a non-Q wave MI. Of 392 patients who sustained an MI according to the new ESC/ACC guidelines for diagnosis of MI, 83 (21\%) had a peak CK <390 IU/l. This increased the incidence of MI in the study population by $27 \%$. This finding is consistent with previous reports. ${ }^{2}{ }^{3}$

The correlation seen between the two biochemical markers seemed stronger in the subgroup of patients who had a peak CK $>390$ IU/l $(R=0.54)$ than in the group of patients whose peak CK was $<390 \mathrm{IU} / \mathrm{l}(R=0.33)$, although the relationships seen in both these groups reached statistical significance. The correlation between TT and peak CK was statistically significant when classified according to different levels of peak CK level. The correlation improved as the level of peak CK increased, indicating that both cardiac markers rise by similar amounts following myocardial damage. In general, a linear relationship exists between increasing concentrations of biochemical markers and the short term mortality in acute MI. ${ }^{5}$ Mortality data were not available for this study group, and a follow up study is currently underway to further explore these data.

\section{Authors' affiliations}

S G Williams, M Jackson, Cardiothoracic Centre, Liverpool, UK and Department of Cardiology, Arrowe Park Hospital, Wirral, UK

\section{REFERENCES}

1 Joint European Society of Cardiology/American College of Cardiology Committee. Myocardial infarction redefined: a consensus document of the joint European Society of Cardiology/American College of Cardiology Committee for the redefinition of myocardial infarction. Eur Heart $J$ 2000;21:1502-13.

2 Ferguson JL, Beckett GJ, Stoddart $M$, et al. Myocardial infarction redefined: the new ACC/ESC definition, based on cardiac troponin, increases the apparent incidence of infarction. Heart 2002;88:343-7.

3 Pell JP, Simpson E, Rodger JC, et al. Impact of changing criteria on incidence, management, and outcome of acute myocardial infarction: retrospective cohort study. BMJ 2003;326:134-5.

4 World Health Organisation Expert Committee. Hypertension and coronary heart disease: classification and criteria for epidemiological studies. Technical report series No 168. Geneva: World Health Organisation, 1959.

5 Ohman EM, Armstrong PW, Christenson RH, et al. Cardiac troponin T levels for risk stratification in patients with myocardial infarction. N Engl J Med 1996;335:1333-41. 